PROCEEDINGS OF THE

AMERICAN MATHEMATICAL SOCIETY

Volume 127, Number 6, Pages 1855-1861

S 0002-9939(99)05194-1

Article electronically published on February 5, 1999

\title{
BOUSFIELD LOCALIZATIONS OF CLASSIFYING SPACES OF NILPOTENT GROUPS
}

\author{
WILLIAM G. DWYER, EMMANUEL DROR FARJOUN, AND DOUGLAS C. RAVENEL \\ (Communicated by Ralph Cohen)
}

\begin{abstract}
Let $G$ be a finitely generated nilpotent group. The object of this paper is to identify the Bousfield localization $L_{h} B G$ of the classifying space $B G$ with respect to a multiplicative complex oriented homology theory $h_{*}$. We show that $L_{h} B G$ is the same as the localization of $B G$ with respect to the ordinary homology theory determined by the ring $h_{0}$.
\end{abstract}

\section{INTRODUCTION}

Let $G$ be a finitely generated nilpotent group. The object of this paper is to identify the Bousfield localization $L_{h} B G$ of the classifying space $B G$ with respect to a multiplicative complex oriented homology theory $h_{*}$. We show that $L_{h} B G$ is the same as the localization of $B G$ with respect to the ordinary homology theory determined by the ring $h_{0}$. This is similar to what happens when one localizes a space $X$ with respect to a connected ring theory $E$ : it follows from results of Bousfield [Bou79, Theorem 3.1] that $L_{E} X$ is the localization of $X$ with respect to ordinary homology with coefficients in the ring $E_{0}$. The point in this paper is that we do not require the spectrum $h$ to be connected.

Our main result is

Theorem 1. Let $G$ be a finitely generated nilpotent group, and let $h_{*}$ be a multiplicative complex oriented homology theory. Then $L_{h} B G=L_{R} B G$, where $R$ is the ring $h_{0}$ and $L_{R}$ is localization with respect to the ordinary homology theory determined by $R$.

The hypothesis that $h_{*}$ be multiplicative is not essential to any of our arguments. We include it mainly to avoid cumbersome statements, and because most complex oriented theories of interest, such as Morava K-theory, are multiplicative. We leave to the interested reader the details of modifying the results here to remove the assumption that $h_{*}$ is multiplicative. Complex orientability is used in an essential way, in the proof of Theorem 3.

Our method of proof is to begin with finite $p$-groups and proceed by induction on the order of the group. We show that if $G$ has a normal subgroup $H$ such that $B H$

Received by the editors September 17, 1997.

1991 Mathematics Subject Classification. Primary 55N20, 55R35.

All three authors were partially supported by the US-Israel Binational Science Foundation, and the first and third authors by the National Science Foundation.

(C)1999 American Mathematical Society 
is $h_{*}$-local, $G / H=\mathbf{Z} / p$, and $B(G / H)$ is $R$-local for $R=h_{0}$, then $B G$ is $h_{*}$-local. We do this by studying the fibration

$$
B H \longrightarrow B G \longrightarrow B(G / H)=B \mathbf{Z} / p \text {. }
$$

To pass to arbitrary finitely generated nilpotent groups, we use the arithmetic square decomposition of $L_{h} X$ due to Mislin and Bousfield.

Our main task is to study the fibration displayed above. In general it is not true that if the base and fibre of a fibration are local with respect to some homology theory, then the total space is also local. For example in the fibration

$$
S^{2} \longrightarrow \mathbf{R} P^{2} \longrightarrow B \mathbf{Z} /(2)
$$

both fibre and base are local with respect to ordinary integral homology, but the total space is not [BK72], [DDK77].

Our technique for dealing with this problem is to use the following lemma. If $B$ is a space and $\mathcal{C}$ is a class of fibrations over $B$, say that $\mathcal{C}$ has $h_{*}$-accessible fibres if any $h_{*}$-equivalence (over $B$ ) between fibrations in $\mathcal{C}$ induces an $h_{*}$-equivalence on fibres.

Lemma 2. Let $h_{*}$ be an arbitrary homology theory, and consider the diagram

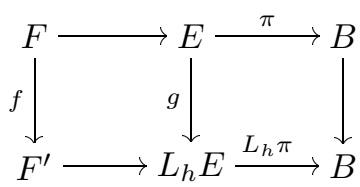

in which each row is a fibre sequence. Suppose that $B$ and $F$ are both $h_{*}$-local, and that there exists some class $\mathcal{C}$ of fibrations over $B$ which has $h_{*}$-accessible fibres and contains both $\pi$ and $L_{h} \pi$. Then $E$ is $h_{*}$-local.

Proof. Since $\mathcal{C}$ has $h_{*}$-accessible fibres and $h_{*}(g)$ is an equivalence, $h_{*}(f)$ is also an equivalence. The space $F$ is $h_{*}$-local by assumption, and $F^{\prime}$ is $h_{*}$-local since it is the homotopy fibre of a map between $h_{*}$-local spaces. Therefore $f$, being an $h_{*}$-equivalence between $h_{*}$-local spaces, is an equivalence. It follows that $g$ is also an equivalence and $E$ is $h_{*}$-local.

In order to use this lemma, we show in the next section that if $h_{*}$ is a multiplicative complex oriented homology theory with the property that $h_{0}$ is a vector space over $\mathbf{Z} / p$, then the class of all fibrations over $B \mathbf{Z} / p$ has $h_{*}$-accessible fibres.

\section{Fibrations over $B \mathbf{Z} / p$}

In this section we prove the following theorem. The results and arguments are inspired by the work of Kriz [Kri].

Theorem 3. Let $h_{*}$ be a multiplicative complex oriented homology theory such that the ring $h_{0}$ is a mod $p$ vector space. Suppose that $E$ and $E^{\prime}$ are fibrations over $B \mathbf{Z} / p$ with fibres $F$ and $F^{\prime}$, respectively, and that $f: E \rightarrow E^{\prime}$ is a map over $B \mathbf{Z} / p$ which induces an isomorphism $h_{*} E \cong h_{*} E^{\prime}$. Then $f$ also induces an isomorphism $h_{*} F \cong h_{*} F^{\prime}$.

Recall that the homotopy coequalizer of a pair of maps $f, g: X \rightarrow Y$ is obtained by taking the cylinder $X \times[0,1]$ and gluing one end to $Y$ by $f$ and the other end to $Y$ by $g$. This construction is sometimes also called the double mapping cylinder of $f$ and $g$. Given maps of pairs $f, g:(X, A) \rightarrow(Y, B)$, the homotopy coequalizer 
of $f$ and $g$ is the pair $(Z, C)$, where $Z$ is the homotopy coequalizer of the two maps $X \rightarrow Y$ and $C$ is the homotopy coequalizer of the two maps $B \rightarrow C$. A diagram of pairs equivalent to

$$
(X, A) \stackrel{f, g}{\longrightarrow}(Y, B) \longrightarrow(Z, C)
$$

is said to be a homotopy coequalizer diagram. The following lemma is elementary.

Lemma 4. Let

$$
(X, A) \stackrel{f, g}{\longrightarrow}(Y, B) \longrightarrow(Z, C)
$$

be a homotopy coequalizer diagram. Suppose that $h_{*}$ is a homology theory. Then there is a natural long exact sequence

$$
\cdots \longrightarrow h_{i}(X, A) \stackrel{f_{*}-g_{*}}{\longrightarrow} h_{i}(Y, B) \longrightarrow h_{i}(Z, C) \longrightarrow h_{i-1}(X, A) \longrightarrow \cdots
$$

Lemma 5. Let $G$ be a group of order $p$ with generator $g$, and $V$ a $\bmod p$ vector space with an action of $G$. Then the endomorphism $(1-g)$ of $V$ is nilpotent (in the sense that for some integer $\left.k,(1-g)^{k}=0\right)$. In particular, the kernel of $(1-g): V \rightarrow V$ is nontrivial.

Proof. It is possible to choose $k=p$, since, in view of the fact that we are working $\bmod p,(1-g)^{p}=1-g^{p}=0$.

Lemma 6. Suppose that $h_{*}$ is a multiplicative complex oriented homology theory. Consider a homotopy fibre square

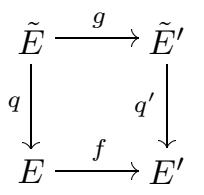

in which $q$ and $q^{\prime}$ are principal $S^{1}$-bundles. If $f$ induces an isomorphism on $h_{*}$, then so does $g$.

Proof. Let $\xi^{\prime}$ be the complex line bundle over $E^{\prime}$ associated to $q^{\prime}$, and $\xi$ the complex line bundle over $E$ associated to $q$. Denote the Thom spaces of these bundles by $M(\xi)$ and $M\left(\xi^{\prime}\right)$ respectively. There is a map of cofibration sequences

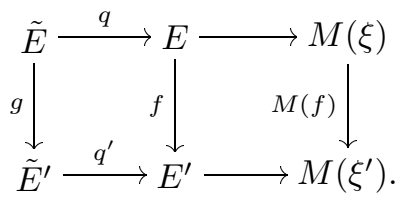

The map $h_{*}(f)$ is an isomorphism by hypothesis. By the Thom isomorphism for $h_{*}$, the map $h_{*}(M(f))$ can be identified with $h_{*}(f)$ and so it too is an isomorphism. The fact that $h_{*}(g)$ is an isomorphism follows from looking at long exact homology sequences and using the five lemma.

Proof of Theorem 3. Let $G$ denote the group $\mathbf{Z} / p$, and $g \in G$ some chosen generator. We can assume that $F$ and $F^{\prime}$ are $G$-spaces, and that $f$ is obtained up to homotopy by taking the Borel construction $\beta(f)$ on a $G$-map $F \rightarrow F^{\prime}$. (One way to obtain a suitable $G$-space equivalent to $F$, for instance, is to take the pullback over $E \rightarrow B G$ of the universal cover of $B G$.) 
Suppose that $X$ is a $G$-space (in our case either $F$ or $F^{\prime}$ ). Note that the homotopy coequalizer of the $G$-maps $1, g: G \rightarrow G$ is the circle $S^{1}$ with the usual rotation action of $G$. More generally, the homotopy coequalizer of $1, g: X \times G \rightarrow X \times G$ is the product $G$-space $X \times S^{1}$. Taking Borel constructions gives a homotopy coequalizer diagram

$$
\beta(X \times G) \stackrel{u, v}{\longrightarrow} \beta(X \times G) \longrightarrow \beta\left(X \times S^{1}\right)
$$

where $u$ and $v$ are the appropriate induced Borel construction maps. It is clear that $\beta\left(X \times S^{1}\right)$ is the total space of a principal $S^{1}$-bundle over $\beta(X)$, in fact, the total space of the pullback along the map $\beta(X) \rightarrow B G$ of the usual principal $S^{1}$ bundle $\beta\left(S^{1}\right) \rightarrow B G$. The Borel construction $\beta(X \times G)$, on the other hand, can be identified up to homotopy with $X$ itself in such a way that $u$ and $v$ can be identified with the original maps 1 and $g$.

Let $\tilde{E}$ denote the Borel construction $B\left(G, F \times S^{1}\right)$ and $\tilde{E}^{\prime}$ the Borel construction $B\left(G, F^{\prime} \times S^{1}\right)$. According to the above considerations we have a homotopy coequalizer diagram

$$
\left(F^{\prime}, F\right) \stackrel{1, g}{\longrightarrow}\left(F^{\prime}, F\right) \longrightarrow\left(\tilde{E}^{\prime}, \tilde{E}\right)
$$

Since $h_{*}\left(E^{\prime}, E\right)$ vanishes by assumption, $h_{*}\left(\tilde{E}^{\prime}, \tilde{E}\right)$ vanishes by Lemma 6 . Lemma 4 then implies that the endomorphism $\left(1-g_{*}\right)$ of $h_{*}\left(F^{\prime}, F\right)$ is an automorphism, but by Lemma 5 this can happen only if $h_{*}\left(F^{\prime}, F\right)=0$, in other words, only if the map $F \rightarrow F^{\prime}$ induces an $h_{*}$-isomorphism.

\section{LOCALIZATION OF CLASSIFYING SPACES}

We begin by recalling a result of Bousfield [Bou82] about localizations of $B \mathbf{Z} / p$. (In that paper he actually determines $L_{h} K(A, n)$ for any homology theory $h_{*}$ and any abelian group $A$.) We say that a space is $h_{*}$-acyclic if the reduced homology $\tilde{h}_{*}(X)$ vanishes, or equivalently if $L_{h}(X)$ is contractible.

Lemma 7. If $h_{*}$ is a multiplicative homology theory, then the space $B \mathbf{Z} / p$ is $h_{*}$ acyclic if $p$ is invertible in $h_{0}$ and $h_{*}$-local otherwise. Equivalently, $L_{h} B \mathbf{Z} / p=$ $L_{R} B \mathbf{Z} / p$, where $R=h_{0}$ and $L_{R}$ denotes localization with respect to $H_{*}(-; R)$.

With Theorem 3 in hand we can prove the following.

Theorem 8. Suppose that $h_{*}$ is a multiplicative complex oriented homology theory, and that $G$ is a finite p-group. The space $B G$ is $h_{*}$-acyclic if $p$ is invertible in $h_{0}$ and $h_{*}$-local otherwise.

Proof. If $p$ is invertible in $h_{0}$, it is obvious from the Atiyah-Hirzebruch spectral sequence that $B G$ is $h_{*}$-acyclic, so we assume that $p$ is not invertible in $h_{0}$ and prove that $B G$ is $h_{*}$-local. Suppose first that $h_{0}$ is a $\mathbf{Z} / p$-vector space. We argue by induction on the order of $G$. Let $H \subset G$ be a normal subgroup of index $p$. The space $B \mathbf{Z} / p$ is $h_{*}$-local by Lemma 7 , and so there is a diagram of fibration sequences:

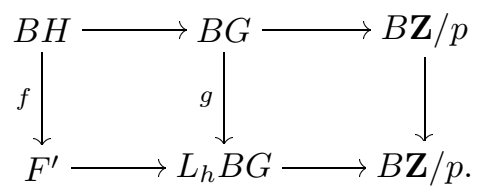


It thus follows immediately from Lemma 2 and Theorem 3 that $B G$ is $h_{*}$-local.

Now consider a general $h$ of the specified type. For a prime $q$, let $h / q$ denote the smash product of the spectrum $h$ representing $h_{*}$ with a mod $q$ Moore spectrum denoted here by $M$. We claim that $h / q$ is still complex orientable: A complex orientation for a spectrum $E$ is a class $x \in E^{2}\left(C P^{\infty}\right)$ with certain properties. One has a map $E \rightarrow E \wedge M$ induced by the unit in $M$, and one can use the image of $x$ under this map as a complex orientation for $E \wedge M$.

Alternatively, $E$ is complex orientable iff it is an $M U$-module spectrum. If $E$ is an $M U$-module spectrum, so is $E \wedge M$.

If $X$ is a space, let $X_{\mathbf{Q}}$ denote the localization of $X$ with respect to rational homology. Since $(B G)_{\mathbf{Q}}=*$, it follows from Proposition 7.2 of [Bou82] that we have a fibration sequence

$$
L_{h} B G \longrightarrow \prod_{q} L_{h / q} B G \longrightarrow\left(\prod_{q} L_{h / q} B G\right)_{\mathbf{Q}}=\text { pt. }
$$

where $q$ runs though the primes not invertible in $h_{0}$. We know from above that $L_{h / p} B G=B G$ and that $L_{h / q} B G=$ pt. for $q \neq p$. It follows that $L_{h} B G=B G$ as claimed.

Slightly more generally we have

Theorem 9. Suppose that $G$ is a finite nilpotent group and that $h$ is a multiplicative complex oriented homology theory. Then $L_{h} B G=L_{R} B G$, where $L_{R}$ is as in Lemma 7. In particular, if no prime dividing the order of $G$ is invertible in $h_{0}$, then $B G$ is $h_{*}$-local.

Proof. The group $G$ is the direct product of its Sylow $p$-subgroups $G_{p}$, so we have $B G \simeq \prod_{p} B G_{p}$ and $L_{h} B G \simeq \prod_{p} L_{h} B G_{p}$. The factors in this second product can be identified with the help of Theorem 8 . There is a similar product formula for $L_{R} B G$.

We now turn to the proof of the main theorem.

Proof of Theorem 1. It is shown by Bousfield in [Bou82] that for any space $X$, $L_{h} X \simeq L_{h} L_{R} X$ where $L_{R}$ is localization with respect to $H_{*}(-; R)$. It is easy to check that a map of spaces is an isomorphism on $H_{*}(-; R)$ if and only if it is an isomorphism on $\bigoplus_{p} H_{*}(-; \mathbf{Z} / p \otimes R)$ as well as an isomorphism on $H_{*}(-; \mathbf{Q} \otimes R)$. Let $\mathcal{P}$ be the set of all primes which are not invertible in $R$. It follows that a map of spaces is an isomorphism on $H_{*}(-; R)$ if and only if it is an isomorphism on $H_{*}\left(-; \bigoplus_{p \in \mathcal{P}} \mathbf{Z} / p\right)$, as well as, if $\mathbf{Q} \otimes R \neq 0$, an isomorphism on $H_{*}(-; \mathbf{Q})$. Since $B G$ is a nilpotent space, the results of [DDK77] imply that if $\mathbf{Q} \otimes R=0$ there is an equivalence

$$
L_{R} B G \simeq \prod_{p \in \mathcal{P}} L_{\mathbf{Z} / p} B G,
$$

while if $\mathbf{Q} \otimes R \neq 0$ there is a homotopy fibre square

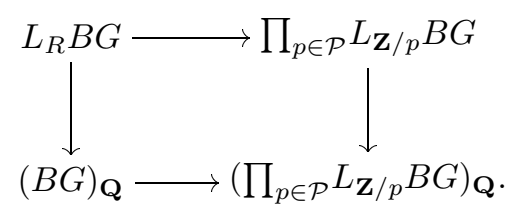


We will carry out the proof by showing that $L_{R} B G$ is $h_{*}$-local, so that $L_{h} B G \simeq$ $L_{h} L_{R} B G \simeq L_{R} B G$. To do this we will show that all of the constituents in the above formulas for $L_{R} B G$ are $h_{*}$-local, and then appeal to the fact that the class of $h_{*}$-local spaces is closed under homotopy inverse limit constructions.

Now according to [BK72, VI 2.6, 2.2 and IV $\S 2]$ the space $L_{\mathbf{Z} / p} B G \simeq(\mathbf{Z} / p)_{\infty} B G$ can be identified as $B\left(G_{p}\right)$, where $G_{p}=\lim G / \Gamma_{s}^{p} G$ is the $p$-lower-central-series completion of $G$. In particular $L_{\mathbf{Z} / p} B G$ is equivalent to the homotopy inverse limit of the tower $\left\{B\left(G / \Gamma_{s}^{p} G\right)\right\}_{s}$. If $p \in \mathcal{P}$, then each space in this tower is $h_{*}$-local (Theorem 8 ), and so $L_{\mathbf{Z} / p} B G$ is $h_{*}$-local by homotopy inverse limit closure of the class of local spaces. By the same principle, $\prod_{p \in \mathcal{P}} L_{\mathbf{Z} / p} B G$ is $h_{*}$-local.

We can complete the proof by showing that if $\mathbf{Q} \otimes R \neq 0$, then any space $W$ local with respect to rational homology is also local with respect to $h_{*}$. Given the definition of what it means for a space to be $h_{*}$-local, we have to show that any $h_{*}$-equivalence $f: X \rightarrow Y$ induces a bijection $f^{\#}:[Y, W] \rightarrow[X, W]$ (where the brackets indicate homotopy classes of maps). However, by [Bou82, 3.3], such an $f$ is a rational equivalence, so the fact that $f^{\#}$ is a bijection follows from the fact that $W$ is local with respect to rational homology.

\section{Possible extensions And Related Problems}

It was shown above that the Bousfield localization with respect to certain homology theories of the classifying space $B G$ of a finitely generated nilpotent group is the same as the localization with respect to a classical homology theory with appropriate coefficients. The question remains open for other (nonfinitely generated) nilpotent groups and other localization functors. Using the fact that $K(F, 2)$, where $F$ is any free abelian group, is local with respect to complex $K$-theory it is not hard to see that so is $K(G, 1)$ for any abelian group $G$ and in fact one can show that Theorem 1 holds for any abelian group.

To go beyond Eilenberg-Mac Lane spaces the following is a natural possible extension of the main results above.

Let $N$ be a nilpotent space whose homotopy groups vanish above certain dimension $n$. Is it true that any Bousfield homological localization of $N$ is equivalent to its localization with respect to a well chosen classical homology theory? More generally the same should be true for an arbitrary polyGEM.

Similar questions arise beyond the realm of Bousfield homological localization. Namely, one may ask for analogues of the above questions for an arbitrary homotopical localization $L_{f}$ with respect to an arbitrary map $f$. In that case it is not true that the localization will be the same as the localization with respect to a well chosen classical homology. This is because the map $B \mathbf{Z} / p^{2} \rightarrow B \mathbf{Z} / p$ is in fact a homotopy localization map, but it is not a homological localization map. But one does expect that an arbitrary localization of a nilpotent space $N$ as above will also be a nilpotent space with vanishing homotopy groups above a certain dimension that depends only on $n$.

\section{REFERENCES}

[BK72] A. K. Bousfield and D. M. Kan. Homotopy Limits, Completions and Localizations, volume 304 of Lecture Notes in Mathematics. Springer-Verlag, 1972. MR 51:1825

[Bou79] A. K. Bousfield. The localization of spectra with respect to homology. Topology, 18:257281, 1979. MR 80m:55006 
[Bou82] A. K. Bousfield. On homology equivalences and homological localizations of spaces. American Journal of Mathematics, 104:1025-1042, 1982. MR 84g:55014

[DDK77] W. G. Dwyer, E. Dror, and D. M. Kan. An arithmetic square for virtually nilpotent spaces. Illinois Journal of Mathematics, 21:242-254, 1977. MR 55:11246

[Kri] I. Kriz. Morava K-theory of classifying spaces: some calculations. Topology. 36:12471273, 1997. CMP 97:13

[Kur56] A. G. Kurosh. The Theory of Groups, Volume Two. Chelsea Publishing Company, New York, 1956. MR 18:188f

Department of Mathematics, University of Notre Dame, Notre Dame, Indiana 46556

E-mail address: william.g.dwyer.1@nd.edu

Department of Mathematics, Hebrew University, Jerusalem, Israel

E-mail address: farjoun@math.huji.ac.il

Department of Mathematics, University of Rochester, Rochester, New York 14627

E-mail address: drav@harpo.math.rochester.edu 